FORMATION Formation emploi

Revue française de sciences sociales

132 | octobre-décembre 2015

Enseignement supérieur et mondes économiques : de nouveaux éclairages

\title{
La composition du corps professoral comme reflet du rapport des écoles d'ingénieurs au(x) monde(s) économique(s)
}

The composition of teaching staff as a reflection of the relations between

engineering schools and the economic world

Die Zusammensetzung der Lehrerschaft als Spiegel der Beziehung zwischen den höheren Ingenieurschulen und der/den Wirtschaftswelt/en

La composición del cuerpo docente como reflejo de la relación de las escuelas de ingeniería con el(los) mundo(s) económico(s)

\section{Antoine Derouet}

\section{OpenEdition}

Journals

Édition électronique

URL : http://journals.openedition.org/formationemploi/4540

DOI : 10.4000/formationemploi.4540

ISSN : 2107-0946

Éditeur

La Documentation française

Édition imprimée

Date de publication : 30 décembre 2015

Pagination : $33-50$

ISSN : 0759-6340

Référence électronique

Antoine Derouet, «La composition du corps professoral comme reflet du rapport des écoles

d'ingénieurs au(x) monde(s) économique(s) », Formation emploi [En ligne], 132 | octobre-décembre

2015, mis en ligne le 30 décembre 2017, consulté le 30 octobre 2020. URL : http://

journals.openedition.org/formationemploi/4540; DOI : https://doi.org/10.4000/formationemploi.4540 


\title{
La composition du corps professoral comme reflet du rapport des écoles d'ingénieurs au(x) monde(s) économique(s)
}

\begin{abstract}
Antoine Derouet
Doctorant en sociologie à l'Ecole des Hautes Etudes en Sciences Sociales (EHESS), équipe Professions, Réseaux, Organisations (PRO) du Centre Maurice Halbwachs (CMH). Ses travaux portent sur l'évolution des formations d'ingénieurs françaises et belges au XXè siècle, plus particulièrement sur l'évolution des contenus d'enseignement.
\end{abstract}

Résumé

La composition du corps prefessoral comme reflet du rapport des écoles d'ingénieurs au(x) monde(s) économique(s)

Affirmer que les formations d'ingénieurs entretiennent des relations étroites avec le monde économique est un lieu commun. Néanmoins, si ces relations sont importantes dans la structuration du système d'enseignement et des institutions, il demeure difficile de les caractériser et d'appréhender leurs effets sur les programmes d'enseignement. Cet article envisage ces relations en examinant la composition du corps professoral. A cet effet, il mobilise une perspective socio-historique qui mêle étude de sa construction sociale en tant qu'enjeu des relations entre formations d'ingénieurs et mondes économiques, et analyse monographique des caractéristiques sociales des enseignants.

Mots clés : grande école, enseignement grande école, diplôme d'ingénieur, ingénieur, contenu de formation, relation école-entreprise, enseignant, identité professionnelle, politique de l'éducation, Ecole centrale de Paris

Abstract

The composition of teaching staff as a reflection of the relations between engineering schools and the economic world

It's a commonplace to assure that engineering schools are in close relationship with the economic world. Nevertheless, if these relations are essential in the structuration of academic system and institutions, it's still difficult to describe them and to comprehend their effects of the training programme. This article considers these relations throught a study of the teaching staff composition. The author follows a socio-historical perspective which combine a study of its social construction as a structuring issue of the relationship between engineering schools and the economic world, and a monographic analysis of teachers' social features in one french engineering schools. 
Keywords : elite school, education in elite higher education schools, engineering degree, engineer, training content, school-enterprise relationship, teacher, professional identity, education policy, Ecole centrale de Paris

Journal of Economic Literature: I 23

Traduction : Auteur

Au sein de l'enseignement supérieur français, les formations d'ingénieurs sont de celles dont l'image sociale est couramment associée à une forte " proximité " avec le monde économique. Cette représentation est notamment relayée par les médias et leurs palmarès ${ }^{1}$. Outre que ces formations constituent la principale voie d'accès au groupe professionnel des ingénieurs (Bouffartigue et Gadéa , 1996), les relations qu'elles entretiennent avec les entreprises et le marché du travail apparaissent comme l'une de leurs préoccupations dominantes, régulièrement évoquées par les institutions d'enseignement et les organisations qui leur sont liées ${ }^{2}$. Plus encore, cette orientation du système d'enseignement technique supérieur est érigée en principe cardinal par l'instance de régulation et de certification des formations, la Commission des Titres d'Ingénieurs (CTi) ${ }^{3}$ : ses référentiels témoignent de la transversalité d'un impératif considéré autant comme une ressource dans le fonctionnement des institutions, à travers la gouvernance ou les financements par exemple, que comme un objectif à atteindre par - et pour - les activités de recherche et d'enseignement.

Dans les programmes, ce leitmotiv est particulièrement perceptible lorsque l'on s'intéresse aux enseignements "socio-économiques", catégorie dont les contours varient selon les périodes et les institutions considérées, faisant, selon les cas, référence à des disciplines « institutionnalisées " (économie, droit, sociologie, gestion...) autant qu’à des contenus moins codifiés (culture générale...) ou plus directement liés à des pratiques professionnelles ${ }^{4}$. Ces enseignements, s'ils ne peuvent se réduire aux relations avec le monde écono-

1. Les classements de revues comme l'Étudiant ou Usine Nouvelle attribuent une grande importance aux critères relatifs à ce qu'ils considèrent comme des indicateurs de la proximité des formations avec "l'entreprise ». Quelle que soit la pertinence des critères retenus, on peut penser qu'ils contribuent à légitimer l'idée que la qualité d'une formation se mesure à l'aune de ses " relations " de divers ordres avec les mondes économiques, qu'il s'agisse des sources de financement de l'école, des pédagogies mises en place, des salaires des diplômés ou du nombre d'anciens élèves en activité.

2. Parmi celles-ci, on peut citer la Conférence des Directeurs des Écoles Françaises d'Ingénieurs (CDEFI), la Conférence des Grandes Ecoles (CGE) ou encore les Ingénieurs et Scientifiques de France (IESF, regroupement d'associations d'anciens élèves, de groupements professionnels et d'associations scientifiques et techniques), dont la composition, les intérêts défendus et les modalités empruntées diffèrent cependant.

3. Fondée en 1934, la CTi dresse la liste des formations habilitées à décerner le titre "d’ingénieur diplômé de (...) ». Depuis 1995, elle publie et actualise un ensemble de documents (Références et orientations) destinés à servir de guide aux formations en vue de leur demande d'habilitation.

4. La dénomination des enseignements étant, en soi, un enjeu voire un objet de luttes, notamment parce qu’elle circonscrit les contenus légitimes, nous préférons les regrouper ici sous un vocable générique, quoique 
mique - et n'en n'ont bien évidement pas l'exclusivité au sein des programmes -, en tirent néanmoins partiellement leur légitimité : leur récurrente mise en débat, depuis le début du XXè siècle, est en effet indissociable des volontés de rapprocher les formations d'ingénieurs de l'exercice professionnel, et plus largement du système productif, leur assignant à ce titre une place particulière dans les curricula.

S’interroger sur les rapports qu'entretiennent les formations d'ingénieurs aux mondes économiques au prisme de ces enseignements renvoie à plusieurs phénomènes distincts mais intimement liés. Il peut ainsi s'agir d'examiner les rôles que jouent des agents et des instruments issus des activités économiques dans l'orientation des formations et dans la recomposition de leur corpus de connaissances (Gervais, 2007) ; l'étude peut également porter sur la pénétration et la diffusion, au sein du système productif, de savoirs produits, transmis et expérimentés par l'enseignement supérieur ; enfin, il est possible d'observer plus particulièrement la préparation des élèves à l'exercice de leur future profession et à leur entrée dans le monde économique (Chambard, 2014) - processus souvent associé au terme polysémique de "professionnalisation" (Demazière et al, 2012) -, ce qui recouvre autant l'acquisition de compétences spécifiques qu'une socialisation anticipée à l'environnement de travail.

En écho à ces questionnements, cet article propose de s'intéresser aux enseignants en charge de ces cours. En effet, la définition de ces enseignants, leur sélection et leurs caractéristiques semblent constituer un enjeu à différentes échelles de la " chaîne de production curriculaire " 5 , influant tant sur les contenus que sur la trajectoire des enseignements (Derouet, 2013).

Ceci encourage à étudier parallèlement la légitimation des enseignements socio-économiques et la production de leur corps professoral. Nous postulerons donc que l'essor progressif de ces enseignements dans les programmes est notamment le produit - et le producteur - d'une redéfinition des rapports aux mondes économiques dont les enseignants seraient les vecteurs. Dans cette perspective, s'intéresser aux logiques qui président à la constitution du corps enseignant constitue une porte d'entrée, un filtre ${ }^{6}$, pour rendre compte des rapports entre mondes économiques et formations d'ingénieurs, dont un programme est à la fois le produit et l'initiateur.

insatisfaisant, "d'enseignement socio-économiques ", qui a pour principal avantage de ne pas être couramment utilisé par les acteurs eux-mêmes.

5. A l'instar de Basil Bernstein, on peut définir celle-ci comme les opérations de sélection, de classification, de distribution, de transmission et d"évaluation des savoirs (Bernstein, 1971), perspective qui suppose d'appréhender les curricula comme un construit social à part entière, et non comme un simple reflet de l'évolution des savoirs ou comme le seul produit - et vecteur - de la codification et de la légitimation des connaissances. 6. Cette démarche s'inspire notamment du travail mené par Fabienne Pavis et Marie-Elizabeth Chessel sur la Fondation Nationale pour l'Enseignement de la Gestion des Entreprises (2001). 
Nous aborderons ici ce double questionnement sur une longue période, afin de mettre l'accent sur les redéfinitions des rapports qu'entretiennent les formations d'ingénieurs aux mondes économiques. Néanmoins, les interprétations possibles dans les limites de cet article sont nécessairement réductrices, écrasant les aspérités temporelles, géographiques et institutionnelles au profit d'une réflexion d'ensemble.

Ladite période s'étend de la seconde moitié de la Première Guerre mondiale aux débuts des années 1970, période de reconfiguration du groupe des ingénieurs et de ses formations, marquée notamment par la réorganisation et le développement du système d'enseignement technique supérieur. Présentant une relative continuité temporelle, cette période préfigure, en partie, les évolutions ultérieures, dont elle pose les jalons organisationnels et intellectuels. Elle permet ainsi d'éclairer certains aspects contemporains qui font figure de lieux communs, comme l'assimilation de ces enseignements au développement des compétences des futurs ingénieurs (connaissance de l'environnement de travail, savoirfaire nécessaires à l'exercice du métier, culture d'entreprise...), ou encore une définition du corps enseignant qui mêle connaissance des pratiques professionnelles et intérêt pour le développement de la recherche, notamment sur les questions pédagogiques?

Notre propos portera successivement sur deux échelles de production des curricula. Tout d'abord, nous nous intéresserons aux débats sur la formation socio-économique des ingénieurs qui, $s^{\prime}$ ils existaient avant la Première Guerre mondiale - comme existaient des enseignements de ce type dans certaines écoles -, prennent une importance renouvelée au sortir de celle-ci, à l'aune de la redéfinition des rapports avec le système productif. Expression des rapports de force qui construisent progressivement la répartition des rôles entre les formations d'ingénieurs et les acteurs économiques dans le fonctionnement de l'enseignement technique supérieur, ces débats vont notamment porter sur la définition du "bon enseignant ", faisant de celui-ci et de son profil des facteurs déterminants.

Cette première partie s'appuiera essentiellement sur l'analyse d'une trentaine de revues professionnelles, syndicales ou scientifiques, auxquelles s'ajoutent des ouvrages et des actes de congrès. Ce corpus de première main a été constitué avec le souci de rétablir les pôles antagonistes qui se sont affrontés, en s'appuyant sur des travaux étudiant cette période (Henry, 2012 ; Hugo-Piron, 2014) mais également à partir des sources mêmes, qui renvoient régulièrement aux prises de positions et/ou aux espaces de débats alliés ou concurrents.

Dans un second temps, nous nous intéresserons plus spécifiquement à la trajectoire des enseignements socio-économiques et à la composition de leur corps enseignant, au sein d'une institution en particulier. Il s'agira de mettre l'accent sur la transformation, à une échelle micro-sociale, des relations aux mondes économiques et de la manière dont les

7. Voir Commission des Titres d'Ingénieurs (2012), Analyses et perspectives, pp. 35-37, [En ligne], URL : www.cti-commission.fr/IMG/PDF/analyse et perspectives 2014-03.pdf 
programmes les exposent. Dans ce cadre, le choix de s'intéresser spécifiquement à l'École Centrale de Paris (ECP) ne se justifie pas tant par la représentativité de cette institution que par la place dominante qu'elle occupe au sein des écoles d'ingénieurs. En effet, l'ECP ne peut pas être considérée comme représentative de l'ensemble - hétérogène - des formations d'ingénieurs ; cependant, elle témoigne d'évolutions institutionnelles et éducatives dont elle a souvent été à l'avant-garde, ce qui s'est traduit par son influence et celle de ses membres dans les débats sur l'enseignement technique supérieur. Ainsi, plutôt que de croiser cette monographie avec celles effectuées sur d'autres institutions ${ }^{8}$, nous avons ici choisi de nous concentrer sur une école. En effet, sa trajectoire éclaire des transformations d'ensemble et cette focalisation permet de souligner l'importance des logiques institutionnelles dans l'élaboration des curricula, tout particulièrement l'importance des liens entre une formation et ses anciens élèves, véritables interfaces avec le groupe professionnel et, plus largement, avec le système productif.

Cette seconde partie s'appuiera sur l'étude des archives de cette école, qui a notamment permis de recenser l'ensemble des cours que l'on peut regrouper sous le qualificatif de "socio-économiques ", de constituer une prosopographie de leur corps enseignant mais également de reconstituer les décisions des directions successives, notamment vis-à-vis des recrutements d'enseignants.

C'est à travers ce changement d'échelles que l'on entend initier une réflexion sur ce que le corps enseignant reflète, ce dont il est le nom, mobilisant l'étude de cette population pour saisir plus largement les rapports entre mondes économiques et formations d'ingénieurs, étoffant ainsi le constat de l'orientation "professionnelle ", " industrielle " ou " économique » des programmes.

\section{Définir le corps enseignant pour redéfinir les relations entre système d'enseignement et mondes économiques}

Dans un premier temps, il convient de restituer les dynamiques qui ont contribué à faire du corps enseignant un " enjeu » déterminant des relations entre formations d'ingénieurs et mondes économiques.

Dans la période considérée, on peut schématiquement mettre en évidence deux cycles argumentatifs durant lesquels se sont opérées des redéfinitions du " bon enseignant », qui recoupent peu ou prou l'entre-deux guerres d'une part, et les années 1945 à 1970, d'autre part. Ces débats ont contribué à légitimer ce que l'on peut qualifier d'orientation "profes-

8. En particulier les monographies de l'Ecole des Hautes Études d'Ingénieurs à Lille, l'École Polytechnique de Louvain et l'École Polytechnique de Bruxelles, effectuées parallèlement à celle de l'École Centrale de Paris, dans le cadre d'un travail de thèse. 
sionnelle " de l'enseignement, soit l'intégration de préoccupations issues du monde social, et notamment des activités économiques, dans les objectifs pédagogiques.

Un déplacement fondateur des objectifs assignés aux institutions d'enseignement s'opère au sortir de la Première Guerre mondiale, dans la continuité des mobilisations en faveur de la recomposition des rapports entre sciences et industries engagée depuis le début de la deuxième industrialisation.

S'ils sont motivés par l'horizon d'un usage accru des savoirs scientifiques par l'industrie, sous ses différentes acceptions (Eildeman, 1986), ces débats se situent également au carrefour de profondes transformations de l'enseignement technique, et plus largement du système éducatif français.

Les controverses autour de la réforme du baccalauréat, en 1902, mais aussi, et surtout, le développement de nouvelles formations d'ingénieurs spécialisées et d'instituts techniques annexés aux universités (Grelon, 1989) tendent à remettre en cause la place dominante des écoles supérieures techniques, comme l'École Polytechnique et ses écoles d'application. C'est dans ce contexte que s'opèrent la réorientation et la réorganisation de l'enseignement technique supérieur, au nom d'une transformation de son apport à « l'industrie ».

Menée notamment par Léon Guillet ${ }^{9}$, cette reconfiguration confère au système d'enseignement le rôle d'organiser la distribution des savoirs " établis » et, partant, de réguler les flux de main-d'œuvre. Ceci repose sur une division du travail éducatif entre les institutions d'enseignement technique, afin de répondre à la pluralité des " besoins " industriels en matière $d$ 'agents et d'experts technico-scientifiques.

Comme le propose Léon Guillet dans une conférence à la Société des Ingénieurs Civils de France, en 1916, publiée dans un ouvrage éponyme (Guillet, 1918), les établissements doivent ainsi être catégorisés selon leur fonction vis-à-vis du système productif : les écoles techniques supérieures à l'enseignement encyclopédique sont destinées à la formation de chefs d'industries ; les instituts dépendant des universités sont destinés à transmettre des savoirs scientifiques approfondis à des ingénieurs spécialistes de sciences ingénieriales (chimie, électricité...) ; les écoles indépendantes à vocation technique et pratique sont chargées de former des techniciens de haut niveau dans des branches particulières ; le Conservatoire national des arts et métiers a une double vocation, la promotion sociale ainsi que la production et la transmission d'une recherche à l'usage de l'industrie.

9. Proche d'Henri Le Chatelier (partisant du»rapprochement» des sciences et de l'industrie), Léon Guillet est une personnalité centrale des débats de cette période. Ingénieur de l'Ecole Centrale, il cumule les positions d'enseignant (au Conservatoire national des arts et métiers) et à Centrale (qu'il dirigera de 1923 à 1944), d'industriel engagé dans la rationalisation (notamment chez De Dion Bouton puis Citroën) et d'expert auprès du ministère du Commerce d'Etienne Clémentel durant la guerre, à la demande duquel il rédige la partie consacrée à l'enseignement dans le «Rapport sur l'organisation industrielle de la France » qui pose les jalons de la politique économique au sortir de la guerre. 
Tandis qu'elle reproduit et relégitime la hiérarchie des formations, cette mise en adéquation du système d'enseignement avec l'organisation du travail repose sur la réorientation des programmes, corollaire pédagogique qui se traduit par la priorité accordée à la préparation des ingénieurs à leur pratique professionnelle.

Se distanciant, d'un côté, de l'encyclopédisme savant, incarné par l'Ecole Polytechnique, et de l'autre, de la recherche appliquée développée au sein des instituts universitaires - tout en s'opposant à l'apprentissage proprement technique et professionnel destiné aux seuls ouvriers et contremaitres -, la mise en œuvre de cet objectif mêle deux logiques : d'une part, il s'agit de transmettre aux élèves un " esprit rationnel " leur permettant d'aborder scientifiquement les problèmes industriels; d'autre part, il s'agit de les préparer à l'usage pratique des sciences. Ce projet met ainsi l'accent sur l'intérêt pédagogique des enseignements pour l'intériorisation de dispositions spécifiques à la pratique de l'ingénieur, au détriment d'un ancrage sur les savoirs. ${ }^{10 .}$

Dans cette optique, la définition du corps enseignant constitue un enjeu considérable. les tenants de cette transformation prônent ainsi le recrutement d'individus ayant une expérience de l'industrie qu'ils doivent enseigner, tout en accordant de l'importance à leurs compétences scientifiques : ceci justifie le développement d'institutions spécialisées dans des disciplines ingénériales et motive également la proposition de créer un titre d'ingénieur-docteur. Si cette seconde idée n'aura qu'une postérité très restreinte, elle temoigne toutefois de l'intérêt particulier qu'accordent les défenseurs des "sciences industrielles" au corps enseignant.

S’il ne s'agit plus de choisir un enseignant pour sa seule valeur scientifique, il n'est pas non plus question de recruter massivement des praticiens pour exposer les techniques de l'industrie, considérées comme trop rapidement " périmées ». L'enseignant doit concilier un capital expert (technique ou scientifique) avec un capital pratique conféré par l'objet de l'expertise, ce qui tend ainsi à faire reposer sur l'individu, et plus encore sur son profil, la détermination de l'enseignement.

Les réflexions sur les enseignements socio-économiques qui émergent durant l'entre-deuxguerres s'inscrivent dans la continuité de cette réorientation de l'enseignement, principalement sour l'impulsion d'un groupement créé sur les bases de la doctrine sociale de l'Eglise ${ }^{11}$, l'Union sociale des ingénieurs catholiques (USIC).

10. Les discussions - marginales - sur les enseignements socio-économiques, épousent cette logique : c'est le cas de l'organisation scientifique du travail, dont l'enseignement est alors essentiellement envisagé pour les ingénieurs des écoles dominantes (en raison de la division du travail éducatif en fonction des futures positions professionnelles) et ne vaut pas tant pour la connaissance des méthodes d'organisation que pour sa contribution à l'apprentissage d'un " esprit " rationnel et à sa mise en pratique dans un environnement de travail.

11. Initiée par l'encyclique Rerum Novarum de Léon XIII en 1891, la doctrine sociale de l'Eglise incite les fidèles à investir le terrain social apostolique ; elle aura une grande importance parmi les ingénieurs, sur le plan organisationnel (par la création d'associations puis de syndicats) autant qu'intellectuel (Grelon, 1995). 
Posant les bases de ce qui deviendra le « cadre » après la Seconde Guerre mondiale, l'USIC redéfinit l'ingénieur autour du « rôle social » qu'elle souhaite lui voir jouer dans la division du travail ; ce rôle associe une fonction d'intermédiaire entre patrons et salariés, de garant de la paix sociale par la gestion de la main-d'oeuvre et de ses conditions de vie, ainsi que d'éducateur des classes populaires dans une visée associant apostolat et combat contre le marxisme. C'est dans ce cadre que le regroupement catholique envisage progressivement le développement d'une formation sociale de l'ingénieur, considérée comme la préparation des élèves à l'exercice de leur profession.

Les débats qui permettent d'élaborer ce projet éducatif, au cours des années 1930, attribuent un rôle majeur à l'enseignant et à ses caractéristiques : si la délimitation du corpus de connaissances reste relativement floue, en revanche, il est fréquemment fait référence à l'expertise professionnelle de l'enseignant sur son sujet ainsi qu'à sa "valeur morale ", spécificité du sujet oblige.

Ainsi, dans le compte-rendu de l'enquête sur la formation sociale, menée en 1941-1942 par l'USIC, qui formalise les réflexions conduites dans les décennies précédentes, les auteurs font du profil des enseignants une caractéristique déterminante des cours de « formation générale humaine ", de "formation particulière du chef " et " d'étude spécialisée des éléments de technique sociale " qu'ils souhaitent voir se développer : selon eux, ces enseignements ne doivent pas revêtir une forme théorique mais prendre l'aspect de conférences données par des personnalités ayant une "expérience personnelle forte " (Commission d'étude de l'Usic, 1942).

Cette logique de constitution du corps enseignant est réaffirmée durant les débats des années 1950. Ceux-ci se situent au croisement de plusieurs facteurs de mise en tension des formations d'ingénieurs que sont, schématiquement, les tentatives de réorganisation du système français d'enseignement et de recherche ${ }^{12}$ (Bongrand, 2012 ; Chatriot et Duclert, 2007), l'engouement pour les savoirs "gestionnaires " et les "sciences de l'homme " (Boltanski, 1981) et l'injonction à un resserrement des relations " industries-Universités ».

Ces débats prolongent et accentuent les conceptions de l'enseignement forgées quelques décennies plus tôt, en bénéficiant notamment de l'importante mobilisation des syndicats de cadres. Ceux-ci investissent alors essentiellement la question des enseignements socioéconomiques, dont il n'avait été question que de manière périphérique lors du cycle argumentatif précédent, durant lequel ces cours étaient principalement associés aux ingénieurs d'élite et destinés à une fraction restreinte d'institutions.

12. La décennie d'après-guerre se caractérise par de multiples tentatives de réformes - souvent avortées - du système d'enseignement et de recherche, menées dans un soucis de démocratisation des études, d'accroissement des flux de main-d'œuvre qualifiée ou encore d'amélioration de l'apport des sciences à l'industrie. Généralement symbolisée par le dépôt du Plan Langevin-Wallon en 1947 et la tenue du Colloque de Caen en 1956, cette période est celle d'une intense réflexion intellectuelle qui a posé les bases du système d'enseignement jusqu’à nos jours. 
Poursuivant les engagements d'organisations pionnières qui défendaient un rôle élargi de l'ingénieur durant l'entre-deux-guerres, comme l'USIC, cette mobilisation correspond à une stratégie de repositionnement des ingénieurs dans la division sociale du travail, en tant que " cadres ", et à une reformulation du rôle que les syndicats de cadres attribuent aux formations dans la préparation des futurs professionnels : les enseignements socio-économiques doivent permettre d'anticiper l'arrivée de l'ingénieur dans son futur environnement de travail, constituant ce qu'un syndicat étudiant, la Fédération Nationale des Associations d'élèves en Grandes Ecoles (FNAGE) qualifie alors de "formation générale professionnelle ». Cette revendication vise à distinguer les formations d'ingénieurs des formations universitaires ${ }^{13}$, en en faisant l'avant-garde des relations industries-universités, tout en légitimant la réorientation des programmes et le redéploiement des enseignements socio-économiques, assimilés à des "savoirs de cadres".

Ces débats font écho aux réflexions de mouvements " modernisateurs » engagés en faveur de l'usage de savoirs socio-économiques comme instruments de pouvoir (Guiader, 2003). Ils adossent la transformation de la division du travail alors en cours - notamment du fait de la concentration du capital et du développement des catégories intermédiaires d'encadrement - à une redistribution des savoirs et des expertises.

Ceci s'opère avec pour corollaire ce que l'on peut qualifier de « domestication » des sciences sociales. Comme le suggère une intervention du président de l'Association Française des Relations Publiques, lors d'une journée d'étude sur les relations Industrie-Enseignement, organisée en février 1956 par un groupement central de la dynamique modernisatrice, le Comité National de l'Organisation Française, cette " domestication " s'opère notamment par la disqualification des travaux universitaires, au profit de connaissances produites pour et par le monde économique : "Personnellement, je pense que c'est un domaine dans lequel la liaison de l'enseignement et du secteur privé aurait un rôle essentiel. Car l'apport du secteur privé amènerait à "épurer", dans une certaine mesure, les théories sociologiques qui font l'objet des disputes de chapelles." (Virenque, 1956)

La définition du "bon enseignant » qui émerge durant ce cycle argumentatif coïncide moins avec le praticien stricto-sensu qu'avec un profil intermédiaire de "formateur-praticien " qui se développe alors au sein de la "nébuleuse managériale " (Touchelay et al., 2008), réseau d'organisations et d'acteurs entendant impulser une " rationalisation " de l'économie et des entreprises, notamment à travers la diffusion des savoirs de gestion. Exerçant généralement le métier d'organisateurs-conseils (Henry, 2012), ces "formateurs-praticiens »

13. Parmi les prises de position en ce sens, on peut citer les critiques à l'encontre de l'inadaptation des formations universitaires d'ingénieurs (à la fois trop abstraites et trop «techniques ") contenues dans le rapport sur la "formation des ingénieurs et des cadres techniques moyens » déposé au Conseil Economique le 10 octobre 1953 par Georges Wolff, figure du syndicalisme cadre. Rédigé dans un contexte de normalisation des formations universitaires - devenues pour la plupart des Écoles Nationales Supérieures d'Ingénieurs (ENSI) - qui se traduit par leur alignement sur les principes des écoles techniques supérieures, ce rapport suggère notamment que l'enseignement soit assuré par des ingénieurs. 
gravitent souvent dans la périphérie des organismes de formations complémentaires et des organisations patronales, comme le Centre de recherche sur les chefs d'entreprises (CRC). Ils participent parallèlement aux débats sur la modernisation où ils contribuent à façonner les caractéristiques des nouveaux enseignants à leur propre image, notamment à travers la mise en exergue de "nouveautés " pédagogiques - comme les séminaires de groupe, les études de cas, les jeux de rôles - dont ils se font les experts.

Ce cycle argumentatif cède progressivement la place, à partir des années 1960-1970, à une période "d'institutionnalisation " des débats caractérisés par leur appropriation par le corps professoral en charge des enseignements socio-économiques au sein des écoles et, dans un second temps, par l'académicisation de celui-ci (Blanchard, 2011).

Si le déplacement qui s'opère alors va progressivement minorer l'importance des acteurs extérieurs aux institutions dans l'orientation des programmes, certaines des caractéristiques légitimées notamment par l'accroissement des rapports avec le système productif vont demeurer structurantes : c'est le cas de l'adossement des enseignements socio-économiques à la préparation du futur ingénieur, tant du point de vue de sa pratique professionnelle que de sa position sociale, et l'importance, dans ce cadre, du corps enseignant.

Ainsi, les débats sur l'enseignement des ingénieurs, dominés par les réinterprétations récurrentes de son orientation " professionnelle ", se concentrent moins sur les savoirs légitimes à enseigner que sur les enseignants qui sont légitimes pour enseigner, leur « déléguant » la responsabilité de la production des enseignements.

\section{Quand le corps professoral donne à voir les mondes économiques : I'exemple de l'Ecole Centrale}

Cette conception de l'enseignant comme intermédiaire des rapports entre formations d'ingénieurs et mondes économiques constitue un lieu commun au sein des institutions, comme le suggère la lecture des archives des écoles. Il convient maintenant de s'intéresser aux pratiques au sein des institutions, autrement dit à la composition du corps professoral et à ce qu'elle nous dit des programmes, afin de mettre en relief l'orientation " professionnelle » des curricula, au-delà des seuls intitulés de cours.

Cette partie s'appuie sur l'étude des enseignements socio-économiques présents au sein de l'École Centrale de Paris. Elle n'abordera pas le recrutement proprement dit des enseignants, bien qu'il soit important d'en connaittre les principales logiques que sont, d'une part, la définition de la juridiction professionnelle des ingénieurs (Abbott, 1988) ${ }^{14}$, et,

14. Ajoutons à ces deux dimensions qui relèvent d'une action des décideurs successifs, l'importance de facteurs institutionnels, comme les actions des enseignants - ou futurs enseignants - dans la définition des cours, voire dans le choix de leur successeur ou encore les « obstacles » aux réformes que peuvent constituer 
d'autre part, les stratégies de positionnement de l'institution au sein des écoles de pouvoir, logique liée au positionnement de Centrale parmi les institutions d'enseignement « les plus consacrées" (Bourdieu, 1989). Nous fournirons ici un aperçu de la composition du corps enseignant, en ce qu'elle exprime un rapport particulier de l'école à des mondes économiques successifs ${ }^{15}$.

Dans cet article, le choix a ainsi été fait de souligner les profils qui sont prépondérants, approche qui se veut toutefois relativement exhaustive étant donné le faible nombre d'enseignants sur la période considérée (de 5 en 1921 à 15 en 1970, avec un faible renouvellement). De même, nous avons choisi de donner du relief à ces «idéaux-types » du corps enseignant en évoquant les principales caractéristiques des enseignants qui apparaissent, à chaque période, comme les plus représentatifs de l'ensemble au vu de l'analyse prosopographique. A l'inverse, appuyer cet aperçu sur un dénombrement des différents profils n'aurait que peu de sens, dans la mesure où la recomposition d'un corps enseignant ne s'opère pas d'un seul tenant mais repose sur un processus de substitution progressive des enseignants en place par de nouveaux individus, l'état du corps professoral à un instant donné étant donc le fruit du chevauchement de plusieurs périodes.

Au sortir de la Première Guerre mondiale, l'école entreprend de réformer son cursus afin de donner une cohérence au programme où s'étaient accumulés des enseignements créés jusqu’alors de manière éparse. Cette réforme est menée par Léon Guillet, dont on a évoqué l'importance dans la réorientation "professionnelle " du système d'enseignement.

Entamée à partir de 1923, et se prolongeant jusqu’à la Seconde Guerre mondiale, cette réforme de l'enseignement est adossée à une recomposition du corps enseignant. Apparaissent de nouveaux profils qui se distinguent de la génération précédente d'enseignants socio-économiques, qui était essentiellement composée de professionnels non ingénieurs, comme Faure (comptabilité) ou Jeanningros et Mollet-Viéville (législation industrielle), pour se rapprocher d'une logique d'endo-recrutement qui pré-existait dans les cours "scientifiques et techniques ". A l'image de Louis Danty-Lafrance, qui prend en charge le cours "d'organisation des usines " en 1924, de Frédéric Harlé, qui enseigne la " propriété industrielle " à partir de 1939, ou de Bernard Lafaille qui prend en charge les " conférences sociales » durant la guerre, ceux-ci sont plus souvent des ingénieurs, ont une expérience de l'industrie et y exercent alors en tant qu'ingénieurs-conseils ${ }^{16}$. Ce sont géné-

les processus de renouvellement des enseignants en place.

15. Cette ambition fait écho au travail de Pierre Bourdieu et Luc Boltanski qui avait permis de mieux caractériser l'inscription d'une institution de pouvoir, l'Institut d'Études Politiques de Paris, dans un espace élitaire, notamment en étudiant la composition du corps enseignant (Bourdieu et Boltanski, 1973).

16. La présence d'ingénieurs-conseils dans le corps enseignant n'est toutefois pas réellement nouvelle, même si elle était jusqu'ici plutôt le fait d'enseignants en sciences exerçant une activité annexe dans l'industrie, à l'instar d'Henry Le Chatelier ou Léon Guillet (Henry, 2012). La nouveauté réside dans une extension de ces profils aux enseignements socio-économiques (qui résulte en partie de l'extension des activités de conseil à ces domaines) mais également dans un renversement du rapport entre leur pratique professionnelle, qui est 
ralement des acteurs importants de groupements syndicaux ou associatifs qui entendent structurer leur espace professionnel, notamment dans le domaine émergeant des activités de conseil (Henry, 2012). Ce dernier point est à souligner puisqu'il semble justifier le recrutement, puis l'extension des prérogatives d'Albert Veyrenc, en charge de la " comptabilité industrielle " à partir de 1930 puis de la " comptabilité et législation industrielle " après 1939 : s'il n'est pas ingénieur, c'est une personnalité importante de la profession d'expert-comptable, dont il présidera l'Ordre après-guerre.

Cet ancrage du corps enseignant dans les pratiques professionnelles des ingénieurs se renforce à la Libération, alors que l'institution entend devenir une «école de cadres » et fait des enseignements socio-économiques l'un des vecteurs de ce repositionnement professionnel. Ainsi, le cours de "propriété industrielle » est confié, en 1950, à Michel Samsoen, diplômé de l'ECP (Ecole centrale de Paris) et docteur en droit, tandis que le nouvel enseignement de "géographie économique " - qui deviendra " économie politique et sociale " - est inauguré la même année par Paul Blanchard, également centralien et docteur en droit.

Alimentée par la "nébuleuse managériale ", cette évolution du cursus se traduit parallèlement par une modification des caractéristiques des enseignants ; ces derniers sont marqués par une proximité plus forte avec le patronat, qu'ils participent aux activités d'organismes liés à celui-ci ou qu'ils aient été eux-mêmes dirigeants d'entreprise. Ainsi, le cours "d'organisation des usines " est confié, en 1949, au centralien George Béquart, Président Directeur Général puis administrateur de la Société d'entreprise municipale d'éclairage, et ancien président du principal groupement rationalisateur, le Comité National de l'Organisation Française (CNOF). Illustré par Béquart, cette recomposition du corps enseignant à l'aune des mouvements modernisateurs se traduit également, à la fin des années 1950, par le recrutement, pour l'enseignement de "relations humaines ", de George Nérot, diplômé de Centrale et docteur en droit, alors administrateur délégué de l'Institut Français des Combustibles et de l'Energie après avoir été le secrétaire général de Gaz de France.

Cette " élévation " du statut des enseignants, qui se caractérise également au niveau de leurs diplômes, fait aussi écho à un accroissement de la concurrence au sein de l'espace des écoles préparant à l'accès aux positions de pouvoir des secteurs public ou privé, du fait de l'apparition de nouvelles institutions (ENA - École Nationale d'Administration) et du développement de plus anciennes (Institut d'Études Politiques de Paris, Ecole des Hautes Études Commerciales).

Ceci amène l'Ecole centrale à tenter de se distinguer en attirant à elle des enseignants dont le capital symbolique doit bénéficier à la reconnaissance de l'institution, stratégie qui motive la tentative de profonde réforme des enseignements socio-économiques en 1964. Afin de rehausser le prestige académique de l'école, et notamment de concurrencer Polytechnique

centrale, et la pratique de l'enseignement, qui est annexe (même si, en l'occurrence, Louis Danty-Lafrance deviendra progressivement un "spécialiste » de l'enseignement, n'exerçant plus réellement dans l'industrie). 
dans l'accès à l'ENA, les enseignements de droit et d'économie sont alors confiés à des universitaires reconnus et attachés à une ouverture de leurs enseignements sur le monde économique : ce sera ainsi le cas de Raymond Barre (problèmes économiques), Roger Houin (problèmes de droit des affaires), Gilbert Tixier (droit économique public et privé) et George Vedel (droit public), dont le profil incarne cette réorientation et ses liens avec la concurrence au sein des écoles de pouvoir ${ }^{17}$.

Toutefois, cette réforme apparaît rapidement comme un échec aux yeux de la direction du fait notamment de son faible effet sur les carrières des élèves - et ces enseignements sont progressivement marginalisés par le développement d'enseignements socio-économiques qui se situent plus dans la continuité des décennies précédentes, adossés à des profils d'ingénieurs et d'ingénieurs-conseils, souvent issus de l'ECP.

C'est sous l'impulsion de l'un d'entre eux, Maurice Teper, que le corps enseignant se recomposera à partir de la fin des années 1960, autour du profil de " praticien-formateur ", dont il présente les caractéristiques idéal-typiques ${ }^{18}$. Le développement important des enseignements socio-économiques, à partir de cette période, s'accompagnera de l' "académicisation " progressive du corps enseignant, dont l'économie et, dans une moindre mesure, la gestion, seront les fers de lance au sein de l'école. N'étant pas nécessairement contradictoire avec le renforcement de l'orientation " professionnalisante " (Pavis, 2010 ; Blanchard, 2011), cette " académicisation " s'opérera, dans un premier temps, en grande partie sur la base des «formateurs-praticiens » qui ont investi les cursus : ainsi, jusqu'aux années 1980, l'élévation des qualifications académiques du corps enseignant - avec la multiplication du nombre de docteurs - ne se réalise pas au détriment de l'expérience professionnelle, les docteurs recrutés ayant généralement exercé dans le secteur privé auparavant. Cette transformation s'opérera dans un second temps, essentiellement à partir des années 1990 et selon des rythmes propres à chaque enseignement, par un recrutement

17. George Vedel était professeur de droit public, doyen de la faculté de Paris, initiateur de la réforme des cursus de droit en 1954 et également enseignant à l'Ecole des Hautes Etudes Commerciales (HEC) et à l'École des Mines.

18. Diplômé de l'École Centrale en 1934, il a participé activement à la "nébuleuse managériale " au sortir de la guerre à travers des missions aux États-Unis et son activité au sein d'organisations patronales, notamment comme intervenant de formations complémentaires (Touchelay et al., 2008). Après avoir été ingénieurconseil au cabinet Paul Planus, il est directeur adjoint de la compagnie Bull lorsqu'il devient chargé de l'enseignement d'" organisation et gestion des entreprises » en 1963. Il s'investit de plus en plus dans ses activités d'enseignement, étant parallèlement chargé de cours à l'Institut européen d'administration des affaires (INSEAD), au Centre de préparation des affaires (organisme patronal) ainsi qu'à l'École d'organisation scientifique du travail (qui dépend du CNOF). Sa trajectoire au sein de l'école témoigne également de l'institutionnalisation progressive du corps enseignant, puisqu'il devient, de 1968 à 1976, le délégué du département "mathématiques appliquées et gestion ", puis celui du département "sciences économiques, sociales et humaines ", créé sous son impulsion en 1976. Pour autant, ses caractéristiques montrent que l'institutionnalisation du corps enseignant ne va pas nécessairement de pair avec l'académicisation, la première pouvant s'effectuer en dehors de toute élévation des qualifications académiques (la seconde peut d'ailleurs, à l'inverse, reposer sur l'accroissement du nombre d'intervenants extérieurs et de vacataires). 
centré sur des critères académiques qu'accompagnera le développement de recherches liées aux pratiques professionnelles ou à la pédagogie ${ }^{19}$, tandis que, parallèlement, le nombre d'intervenants extérieurs croîtra fortement dans les cursus, perpétuant la mobilisation de praticiens au sein des cursus.

\section{Conclusion}

Cet aperçu renforce l'hypothèse du rôle déterminant assigné aux enseignants dans l'élaboration des contenus de cours, division du travail curriculaire relativement commune au sein de l'enseignement supérieur. Néanmoins, les justifications et les objectifs de cette autonomie du corps professoral sur le contenu des enseignements different de celles construites à partir du XIXè siècle au sein, notamment, des universités françaises (Musselin, 2001), tout comme diffèrent les caractéristiques attribuées au " bon enseignant ».

En effet, cette répartition des rôles est légitimée par l'établissement de relations privilégiées entre les formations d'ingénieurs et le système productif, entités dont les enseignants sont chargés d'assurer la médiation. Ainsi, la composition du corps professoral revêt-elle une grande importance au sein des débats ; à l'inverse, il est très peu question de la sélection des connaissances, la détermination des contenus étant adossée aux compétences professionnelles des enseignants et, partant, à leurs caractéristiques et aux capitaux qui légitiment leur recrutement, qui se distinguent des qualifications académiques puisqu'ils reposent prioritairement sur la position occupée dans le monde social, plus spécifiquement dans le système productif et dans le groupe professionnel.

Cet adossement de l'orientation " professionnelle " des programmes aux caractéristiques des enseignants est particulièrement perceptible lorsque l'on s'intéresse à la transcription de cette injonction dans les politiques éducatives des institutions.

L'étude du corps professoral de l'Ecole Centrale témoigne particulièrement de cette logique, présentant des générations distinctes de profils qui correspondent aux orientations pédagogiques du moment ; le travail de détermination des enseignements étant moins le fruit d'une sélection des savoirs que la résultante d'une mise en cohérence des caractéristiques de l'enseignant avec des stratégies institutionnelles.

En portant moins sur la valeur " académique » des candidats que sur les attributs qui permettent à l'institution de se positionner dans le monde social, la sélection des enseignants

19. Un ouvrage collectif, paru en 2007 (Faucheux et Forest, 2007), met en lumière le développement de la recherche en sciences humaines et sociales dans les formations d'ingénieurs, ainsi que ses caractéristiques. Par ricochet, cet ouvrage plaide implicitement pour la systématisation de l'adossement de ces enseignements à la recherche, idée également défendue par le réseau Ingénium (qui entend regrouper les enseignants des "sciences de l'homme et de la société » dans les écoles d'ingénieurs) et reprise depuis quelques années, on l'a évoqué, par la Commission des Titres dans ses « Références et orientations ». 
tend à favoriser le recrutement de l'école au sein du groupe de pairs ${ }^{20}$, rendant possible une « actualisation » de l'enseignement qui se calque sur les évolutions des pratiques du groupe.

Le groupe de pairs est d'ailleurs d'abord celui de l'institution, autrement dit ses anciens élèves, avant d'être celui des ingénieurs. Les enseignements ne sont donc pas tant liés " aux » mondes économiques qu’à " un " monde économique délimité ${ }^{21}$, favorisant une forme de maîtrise du groupe de pairs sur la production et la transmission de ses propres savoirs et compétences d'expert ${ }^{22}$, ce qui peut apparaître comme l'une des caractéristiques des groupes professionnels "établis " mise en évidence par la sociologie des professions (Merton et al., 1957; Freidson, 1986).

Ce mode de recrutement et les modalités de relations aux mondes économiques qu'il incarne pourraient a priori sembler fragilisés par la dynamique d'académicisation qui traverse les écoles d'ingénieurs depuis les années 1980, et ce, sous l'effet d'injonctions croissantes à l'élévation des qualifications académiques, tendant à faire du doctorat un standard (Blanchard, 2014). On peut néanmoins tempérer la " rupture " que peut représenter a priori, cette transformation qui, du reste, ne s'effectue pas au même rythme selon la position des institutions dans l'espace des écoles d'ingenieurs. D'une part, cette transformation s'opère dans la continuité d'une conception de l'enseignant qui, si elle insistait sur son ancrage "professionnel ", lui associait également des compétences expertes tant dans le domaine enseigné que sur les questions pédagogiques, ce dont les «formateurs-praticiens » étaient l'archétype. D'autre part, le développement de formations doctorales dans ces établissements peut laisser penser que les recrutements futurs s'opéreront au sein de cette population d'individus ayant un double capital ingénieur/docteur, prolongeant, sous une nouvelle forme, les dynamiques d'endo-recrutement en supplantant progressivement les profils plus « universitaires » recrutés dans l'intervalle.

Si ces questions restent en suspens, tout incite à les appréhender en reconsidérant les frontières et les distinctions entre formations et mondes économiques, et par conséquent

20. L'importance de cet endo-recrutement dans les autres écoles doit cependant être tempérée : on peut penser qu'il est plus fréquent à l'ECP qu'au sein d'institutions moins élevées dans la hiérarchie des écoles d'ingénieurs, dans la mesure où de telles institutions ont tout intérêt à recruter hors de leurs propres diplômés pour rehausser leur pestige, puisant cependant également dans la population des ingénieurs. Cependant, comme le révèle le travail d'Anne Bidois (2003) sur l'école de Chimie de Rouen, l'endo-recrutement est une pratique courante dans la constitution d'un corps enseignant.

21. Le monde économique ainsi circonscrit laisse apparaître les rapports de force au sein de la configuration professionnelle : de fait, si la revendication d'une orientation professionnelle des formations adossées aux enseignements socio-économiques a longtemps été portée par les syndicats d'ingénieurs, ces derniers sont peu représentés dans les corps enseignants.

22. Cet « endo-recrutement " peut être également considéré comme une condition de l'autonomie enseignante sur la détermination et le déroulement de l'enseignement, favorisant la constitution d'un groupe très homogène partageant des valeurs et des conceptions communes, qui permet l'assouplissement des hiérarchies et des relations de pouvoir. 
les rapports entre ces entités, tant on peut voir à travers cet éclairage historique que leurs interpénétrations sont constitutives de leur fonctionnement, ce qu'illustre la valorisation de la multipositionnalité des enseignants.

\section{Bibliographie}

Abbott A. (1988), The System of Professions: An Essay on the Division of Expert Labor, Chicago, University of Chicago Press.

Bernstein B. (1971), "On the classification and framing of Educational knowledge", in Young M. (dir.), Knowledge and control: new directions for the sociology of education, Londres, Collier-Macmillan, 1971, pp. 47-69.

Bidois A. (2003), "La formation des ingénieurs chimistes à Rouen", Formation Emploi, $\mathrm{n}^{\circ} 83$, pp. 65-76.

Blanchard M. (2011), "Développer la recherche et maintenir la professionnalisation ", Cahiers de la rcherche sur l'éducation et les savoirs, Hors-série n 3, pp. 61-76.

Blanchard M. (2014), «Le rôle de la concurrence dans l'essor des formations supérieures de commerce», Formation Emploi n ${ }^{\circ} 125$, pp. 7-28

Bongrand P. (2012), « La mise en système et l'économicisation de l'enseignement en France au début des années 1950 : la fonctionnalisation d'une institution ", Politix, $n^{\circ} 98$, pp. 35-56.

Bouffartigue P. et Gadéa C. (1997), « Les ingénieurs français. Spécificités nationales et dynamiques récentes d'un groupe professionnel ", Revue Française de sociologie, vol. 37 , $\mathrm{n}^{\mathrm{o}} 1$, pp. 301-326.

Bidois, A. (2003), "La formation des ingénieurs chimistes, à Rouen, 1895-1985 ", Formation Emploi, n ${ }^{\circ}$ 83, juillet-septembre, pp. 65-76.

Boltanski L. (1981), "America, America... le plan Marshall et l'importation du "management" ", Actes de la recherche en sciences sociales, vol. 38, n¹, pp. 1941.

Bourdieu P. et Boltanski L. (1976), "La production de l'idéologie dominante », Actes de la recherche en sciences sociales, vol. $2, \mathrm{n}^{\circ} 2-3$, pp. 373 .

Bourdieu P. (1989), La Noblesse d'Etat. Grandes écoles et esprit de corps, Paris, Editions de Minuit.

Chambard O. (2014), "L'éducation des étudiants à l'esprit d'entreprendre: entre promotion d'une idéologie de l'entreprise et ouverture de perspective émancipatrice ", Formation Emploi, n 127, pp. 7-26. 
Chatriot A. et Duclert V. (s.d.) (2007), Le gouvernement de la recherche. Histoire d'un engagement politique, de Pierre Mendès France au général de Gaulle (1953-1969), Paris, La Découverte.

Chessel M.-E et Pavis F. (2001), Le technocrate, le patron et le professeur : une histoire de l'enseignement supérieur de gestion, Paris, Belin.

Commission d'études de l'Usic (1942), «La formation sociale de l'élève-ingénieur », Echo de l'USIC, juin-juillet-août pp. 230-237.

Demazière D., Roquet P., Wittorski R. (s.d.) (2013), La professionnalisation mise en objet, Paris, L'Harmattan.

Derouet A. (2013), "De l'honnête homme au manager ? La contribution des enseignements juridiques de l'Ecole Centrale à la définition d'un ingénieur d'élite depuis 1829 ", Droit \& Société, n83, pp. 33-47.

Eidelman J. (1986), "Science industrielle contre science pure : la professionnalisation de la recherche dans les années trente ", in Grelon A. (s.d.), Les ingénieurs de la crise: titre et profession entre les deux guerres, Paris, Édition de l'EHESS, pp. 113-116.

Faucheux M. et Forest J. (s.d.) (2007), Les recherches en sciences humaines et sociales dans les écoles d'ingénieurs, Paris, Edition Pétra.

Freidson E. (1986), Professionnal Powers: A study of the Institutionalization of formal Knowledge, Chicago, Chicago University Press.

Gervais J. (2007), La réforme des cadres de l'action publique ou la fabrique d'un "nouveau " corps des Ponts et Chaussées. Impératifs managériaux, logiques administratives et stratégies corporatistes (fin du XIXème siècle), Thèse de doctorat en science politique, IEP de Lyon.

Grelon A. (1989), "Les universités et la formation des ingénieurs en France (18701914)», Formation Emploi, n ${ }^{\circ} 27-28$, pp. 65-88.

Grelon A. (1995), "L'ingénieur catholique et son rôle social », in Cohen Y. et Baudouï R. (s.d.), Les chantiers de la paix sociale (1900-1940), Fontenay/Saint-Cloud, ENS Editions, pp. 167-184.

Guiader V. (2002), « Gaston Berger, un promoteur multipositionnel des sciences sociales (1953-1960) ", Guiader V. et Defaud N. (s.d.), Discipliner les sciences sociales. Les usages sociaux des frontières scientifiques, Paris, L'Harmattan, pp. 47-77.

Guillet L. (1918), L'enseignement technique supérieur à l'après-guerre, Paris, Payot.

Henry O. (2012), Les guérisseurs de l'économie : Sociogenèse du métier de consultant, Paris, CNRS Édition.

Hugot-Piron N. (2014), Les " cadres âgés ». Histoire d'une catégorie de chômeurs, Rennes, Presses Universitaires de Rennes. 
Merton R.K., Reader G.G., Kendall P. (s.d.) (1957), The Student Physician, Introductory studies in the sociology of medical education, Cambridge, Harvard University Press.

Musselin C. (2001), La longue marche des universités françaises, Paris, PUF.

Pavis F. (2003), Sociologie d'une discipline hétéronome. Le monde des formations en gestion entre universités et entreprises en France. Années 1960-1990, Thèse de doctorat en sociologie, université Paris I Panthéon-Sorbonne.

Touchelay B., Druelle-Korn C. et Boulat R. (2008), "Les étapes de la formation d'une « nébuleuse managériale " dans les deux premiers tiers du XXe siècle ", Université Paris Est-Marne la Vallée, [En ligne], URL : http://mtpf.mlab-innovation.net/fr/sommaire/ diffuseurs-de-doctrine,-auteurs-et-dogmes-en-management/les-étapes-de-la-formation.

Virenque (1956), "Journées d'étude industrie-enseignement ", CNOF - revue mensuelle de l'organisation, juin, p. 31. 